The Impact of Information on Attitudes towards E-Mental

\title{
Health Services
}

Running Title: Attitudes towards E-Mental Health Services 


\begin{abstract}
E-mental health services are internet-based treatment options for mental illness. There has been a proliferation of these services in recent years. However, the limited research available indicates that attitudes about e-mental health services are less than optimal. Past research has found that providing information about services can improve attitudes. This study investigated the relationship between knowledge of e-mental health services and attitudes toward e-mental health services. The attitudes examined were the perceived helpfulness of e-mental health services, and the likelihood of using the services. Participants $(N=217)$ were randomly assigned to one of three conditions: provision of emental health information by means of film; provision of e-mental health information by text; or provision of no e-mental health information. Main effects were found for type of e-mental health service and for both perceived helpfulness and likelihood of future use. Participants perceived online programs without therapist assistance as being significantly less helpful, and reported reduced likelihood of engaging these programs when compared to other e-mental health services. There was also a main effect for type of information intervention, in that the text group reported higher likelihood of e-mental health use in the future, whereas there were no effects for the film group. Results indicate that participants perceive important differences between types of e-mental health services, and that a brief text intervention can improve attitudes toward these services. Limitations of the present study and directions for future research are discussed.
\end{abstract}




\section{Introduction}

Relatively little is known regarding consumer attitudes towards e-mental health services. However, the limited research available indicates that consumers who have not yet experienced involvement in these services rate them as unlikely to be particularly helpful, and report low likelihood of using these services in the future ${ }^{1}$. In contrast, consumers that use e-mental health services report them to be an effective and acceptable treatment option ${ }^{2,3}$.

Clearly, it is important to investigate methods of improving attitudes towards emental health. As a relatively new form of treatment, consumers are likely to be unfamiliar with e-mental health services. Yet there is some evidence that suggests they

may be interested in at least learning more about these treatment options ${ }^{4,5}$. Interventions can improve attitudes about mental health services ${ }^{6,7}$. Providing information about the efficacy and format of e-mental health services may have a positive influence upon attitudes, and in turn, increase service usage. However, to date, this possibility has not been investigated.

\section{The Current Study}

The present research investigated whether providing information about e-mental health services might result in higher ratings of perceived helpfulness of services, as well as higher likelihood of future use of the service.

Information conditions were compared to a control condition in which no emental health information was provided. E-mental health service information was provided as either text or film, as is often utilised in advertising and public awareness 
campaigns ${ }^{8,9}$. The information provided was brief, as opposed to the more extensive interventions utilised in previous research ${ }^{6,10,11}$. The current study utilized four service categories (information websites, online counselling, programs with therapist assistance, and programs without therapist assistance) as based on the research of Klein and Cook ${ }^{4}$.

It was hypothesized that provision of educational e-mental health information would be associated with higher ratings of perceived helpfulness and likelihood of future use of these services, when compared to the control condition.

\section{Method}

\section{Participants}

Two hundred and thirty-eight participants completed the online survey. Of these participants, 164 were recruited via email and social networking sites, and 74 were recruited from a first year student research participation pool. Students who took part in the study were awarded partial course credit for their participation. Participants were required to be at least 17 years of age.

Twenty-one participants were excluded (18 = incomplete questionnaire data, 1 = invalid age, 2 = younger than 17 years). The remaining 217 participants consisted of 48 males and 169 females. Of the 217 participants, 70 were randomly assigned to the control group, 66 to the text information group, and 72 participants to the film information group. The age of participants ranged from 17 to 60 years $($ mean $=29.74$, median $=26, S D=$ 11.94).

\section{Design}

The study utilized a mixed factorial design. The independent variables were the information group (three conditions; text, film, control) and type of e-mental health 
service (four levels; information websites, online counselling, online program with therapist assistance, online program without therapist assistance). The dependent variables were attitudes to e-mental health services, as measured by perceived helpfulness of services and likelihood of future use of services.

\section{Materials}

The survey was delivered electronically, by means of online questionnaire software developed by the Research Survey Centre at Griffith University ${ }^{12}$.

Demographic Variables. The demographics variables measured included gender, age in years, ethnic background (nine levels; Asian, Middle Eastern, Pacific Islander, Caucasian, Indian, Indigenous Australian, Multiracial, African, and Other), relationship status (two levels; single, in a relationship), and level of education (three levels; secondary education or less, finished or completing tertiary education, finished or completing postgraduate education).

Perceived Helpfulness of Services. Questions relating to the perceived helpfulness of the services were those used by Klein \& Cook $^{4}$. Participants responded to the question, "How helpful do you think the following services would be if you were if you were going through a personal problem of some sort (e.g., something worrying you, you were going through a tough time)?”

As in Klein \& Cook's ${ }^{4}$ study, 11 types of mental health treatment (both traditional and e-mental health services) were included. Participants rated each service on a seven point scale, ranging from "Very harmful”, to "Very helpful”, with "Neither helpful or harmful” as the neutral centre point on the scale.

Likelihood of Future Use. The measure for likelihood of future use was also 
based on Klein \& Cook's ${ }^{4}$ study. Participants were asked, "If you were going through a personal problem of some sort (e.g., something worrying you, you were going through a tough time), how likely is it that you would use the following services? Please rate your opinion on the following scale”. Eleven forms of mental health service were included. Each service was rated on a seven point Likert scale ranging from "Very unlikely" to "Very unlikely", with "Neither likely nor unlikely" as the neutral centre of the scale.

e-Mental Health Information. An e-mental health information script was developed to explain that treatment for mental health problems can be delivered via the internet. Information was provided relating to online counselling; internet programs with therapist assistance; internet programs without therapist assistance; and information websites. The content focused on describing the different e-mental health services, formats, efficacy and benefits. Also discussed were the types of problems which can be addressed through e-mental health services. The e-mental health service information was provided in two formats.

Film. The film was presented by a 25 year old male actor. The actor presented the e-mental health service information script while looking directly into the camera. The film was 2 minutes and 35 seconds in duration, and was presented against a black background.

Text. The same e-mental health service information was also presented as text.

\section{Procedure}

Participants were provided with a link to the online survey. Participants provided informed consent on the first page of the online survey, by selecting to proceed.

Demographic data was collected. Participants were then randomly allocated to 
one of the three information groups (control, text, or film) by means of the online survey software. Participants allocated to the control group completed the Helpfulness and Likelihood of Future Use measures. Participants allocated to the text or film group viewed the e-mental health information message, and then also completed the Helpfulness and Likelihood of Future Use measures. Within the likelihood and future use scales, the order of presentation of the eleven mental health services was randomly generated for each participant.

\section{Results}

\section{Preliminary Analyses}

Chi-square tests of independence and a one-way ANOVA were run to investigate whether the control, text or film information groups were significantly different on demographic variables.

There were no significant differences between the groups in relation to gender $\left(\chi^{2}(2, N=217)=1.577, p=.455\right.$, Cramer's $\left.V=.085\right)$, ethnicity $\left(\chi^{2}(14, N=217)=\right.$ $17.748, p=.218$, Cramer's $V=.202)$, education $\left(\chi^{2}(4, N=217)=4.527, p=.339\right.$, Cramer's $V=.102)$, relationship status $\left(\chi^{2}(2, N=216)=.398, p=.819\right.$, Cramer's $V=$ $.043)$, or age $\left(F(2,214)=2.197, p=.114, \eta^{2}=.020\right)$.

\section{Assumptions of Main Analyses}

Data was screened for outliers and normality. Where necessary, analyses were run with and without outliers and square root transformations. As there were no substantive differences, the reported analyses are based on untransformed data with outliers included.

\section{Main Analyses}

Two mixed factorial Analyses of Variance (ANOVAs) were conducted using the 
independent variables of type of information and type of e-mental health service. A significance level of $p<.05$ was applied to all analyses. Effect sizes were calculated using eta squared.

Perceived Helpfulness. A Two-way mixed ANOVA was conducted on participants’ scores on perceived helpfulness of services. Mauchley’s test of sphericity was significant $(p=.002)$ indicating that the assumption of covariance had been violated. As such a Greenhouse-Geisser correction was made. The main effect of type of e-mental health service was significant $\left(F(2.836,606.836)=68.887, p<.001, \eta^{2}=.241\right)$. The main effect of type of information was not significant $\left(F(2,214)=2.643, p=.073, \eta^{2}=\right.$ .024), nor was the interaction between information and e-mental health service $(F(5.671$, 606.836) $\left.=1.492, p=.182, \eta^{2}=.010\right)$.

The main effect of type of e-mental health service was followed up with post hoc main effects comparisons using a Bonferroni correction. It was found that the online program without therapist assistance was perceived by participants as being significantly less helpful than the information website $(p<.001)$, online counselling service $(p<.001)$, and online program with therapist assistance $(p<.001)$. Descriptive statistics are displayed in Figure 1.

Likelihood of Future Use. A Two-way mixed ANOVA was conducted on participants' scores on likelihood of future use of services. Mauchley’s test of sphericity was significant $(p<.001)$ indicating that the assumption of covariance had been violated. As such a Greenhouse-Geisser correction was made. The main effect of type of e-mental health service was significant $\left(F(2.714,580.831)=48.946, p<.001, \eta^{2}=.184\right)$, as was the main effect of type of information $\left(F(2,214)=5.280, p=.006, \eta^{2}=.047\right)$. The 
interaction between information and e-mental health service was not significant $(F(5.428$, 580.831) $\left.=1.431, p=.206, \eta^{2}=.011\right)$. These effects are displayed in Figure 2.

The main effect of type of e-mental health service was followed up with post hoc main effects comparisons using a Bonferroni correction. Participants reported a significantly greater likelihood of using information websites than online counselling services $(p<.001)$, or programs with $(p<.001)$ or without $(p<.001)$ therapist assistance. Furthermore, participants reported a significantly lower likelihood of future use of online programs without therapist assistance compared to information websites $(p<.001)$, online counselling $(p=.003)$, or online programs with therapist assistance $(p=.002)$.

The main effect of type of information intervention was also followed up with post hoc main effects comparisons using a Bonferroni correction. Compared to the control group, participants reported significantly greater likelihood of using one of the four e-mental health services when educational information was presented to them in a text format prior to making the rating $(p=.004)$. No significant differences were found between the text and film groups, or film and control groups.

\section{Discussion}

This study investigated whether provision of educational service information could improve consumer attitudes toward e-mental health services. The attitudes of interest in this study were perceived helpfulness of services, and the reported likelihood of future use.

The main effects of information upon the perceived helpfulness and likelihood of future use of e-mental health services were examined. It was found that a brief text 
intervention significantly improved participant ratings of likelihood of future use of emental health services, although not their view on perceived helpfulness of these services. No effects were found for the film intervention on participant attitudes toward e-mental health services.

Although not of primary focus in this study, differences in attitudes toward the four types of e-mental health services was also examined. It was found that regardless of information intervention, online programs with no therapist fared worse than other emental health services. Participants endorsed lower ratings of perceived helpfulness and likelihood of future use for these programs. These results indicate that e-mental health treatments involving therapist support generate more positive attitudes in consumers than those with a self-directed format even prior to actual use. Overall, the findings suggest that consumers perceive important differences between e-mental health services.

Previous research has suggested that gaining knowledge about mental health services can improve consumer attitudes ${ }^{7}$. It has also been suggested that negative attitudes towards e-mental health services are related to a lack of familiarity with these treatment options $5,7,13,14$. The findings of the current study partially support these assertions, as text information was associated with more positive attitudes for e-mental health services. It may be beneficial for future research to examine the efficacy of film information strategies. It is possible that variables such as perceived credibility, authority, or gender may have influenced the effects of this medium of communication. Such information may prove valuable in the designing of e-mental health websites, programs, and advertising.

In summary, this research examined whether the provision of information relating 
to e-mental health services could improve consumer attitudes. A simple text intervention was able to significantly improve attitudes towards e-mental health services. Increasing the usage of e-mental health services will be vital in addressing the unmet needs for mental health treatment, and improving attitudes of consumers towards this form of intervention will play an important role in increasing uptake of these services.

Author Disclosure Statement: No competing financial interests exist 


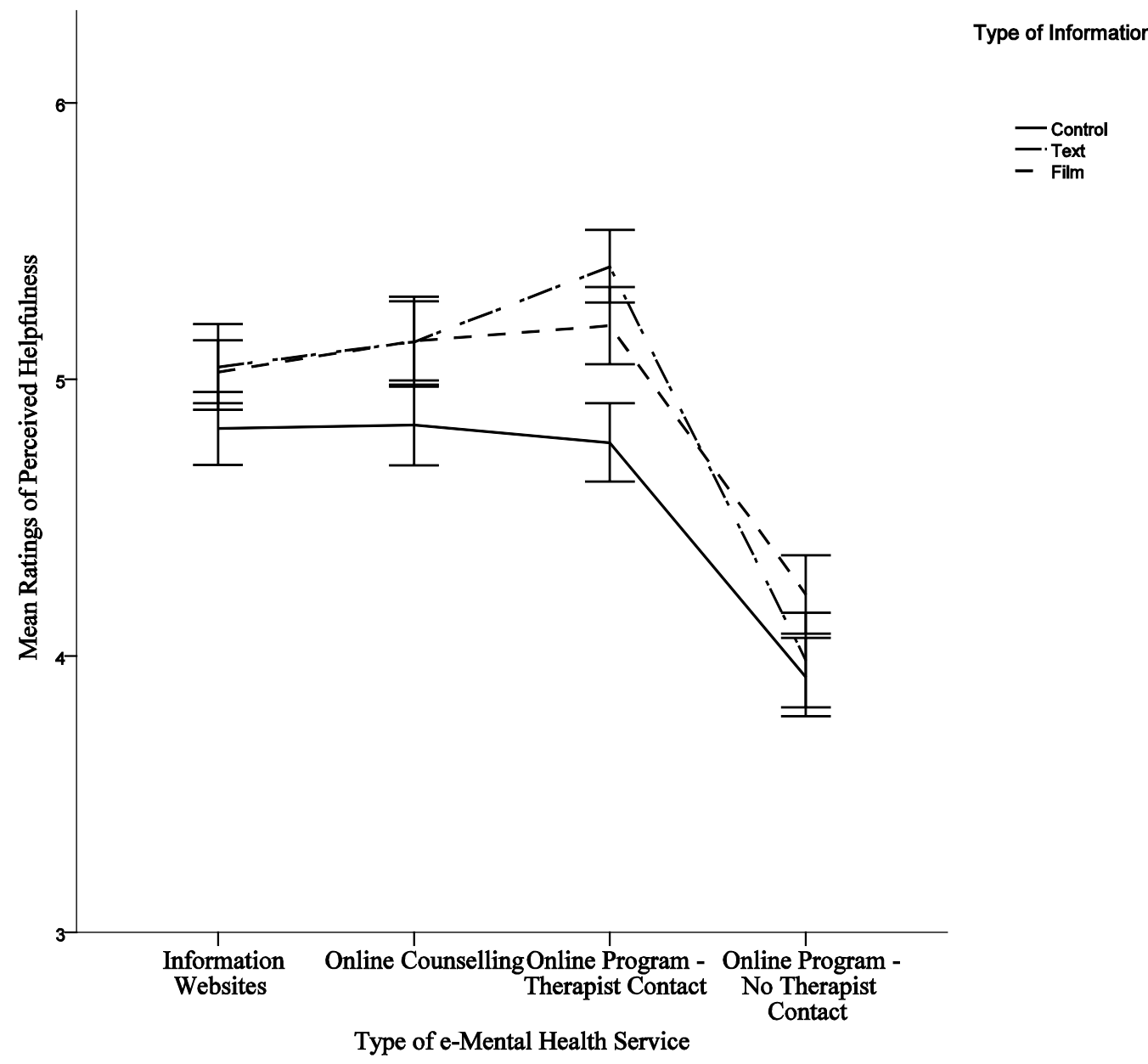

Figure 1. Mean participant ratings of perceived helpfulness of e-mental health services. Error bars represent $+/-1$ standar of the mean. 


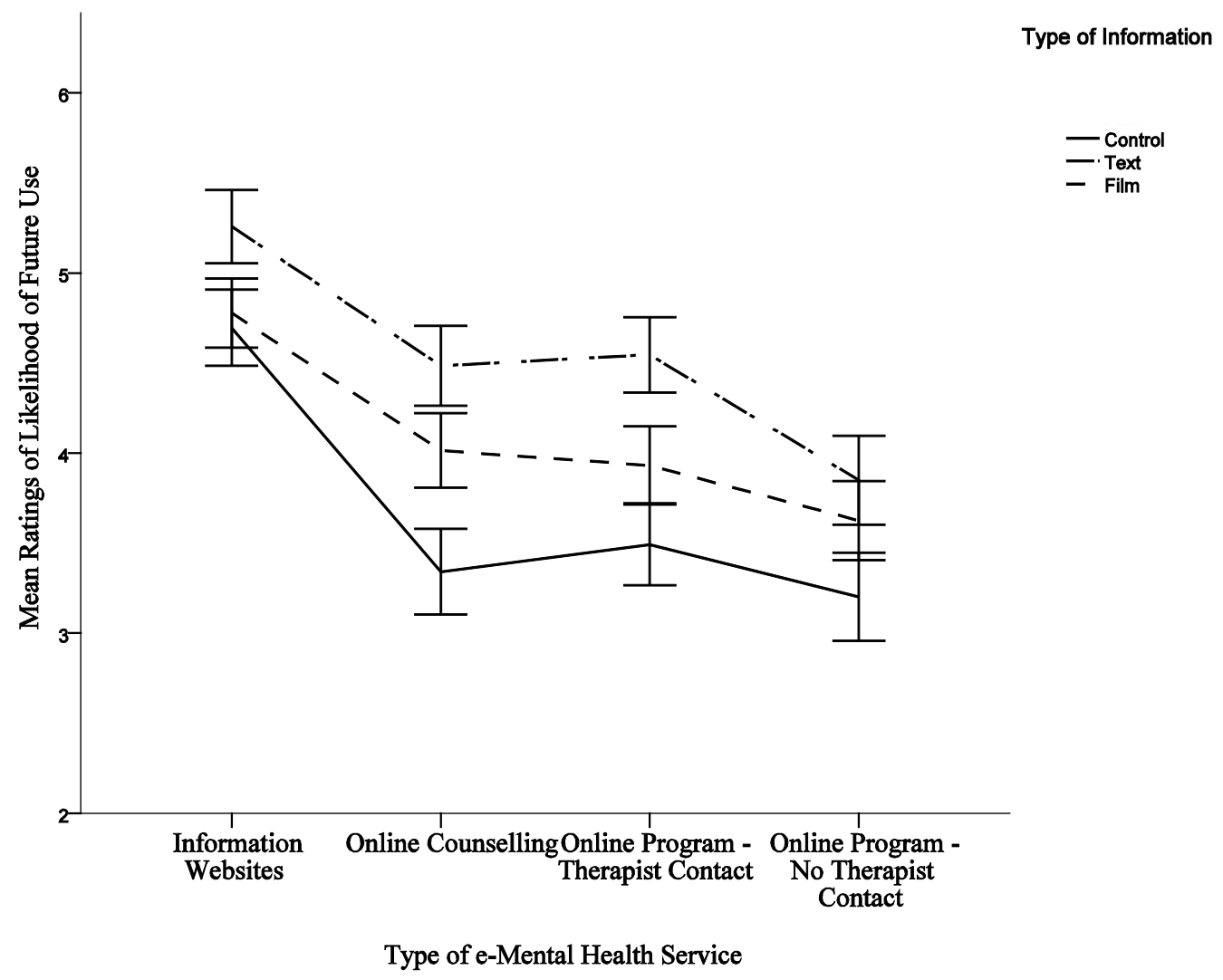

Figure 2. Mean participant ratings of likelihood of future use of e-mental health services. Error bars represent $+/-1$ standa of the mean. 


\section{References}

1. Klein B, Cook S. Preferences for e-mental health services amongst an online Australian Sample. e-Journal of Applied Psychology. 2010;6:28-39.

2. Titov N, Andrews G, Johnston L, Schwencke G, Choi I. Shyness programme: Longer term benefits, cost-effectiveness, and acceptability. Australian \& New Zealand Journal of Psychiatry. 2009;43:36-44.

3. Botella C, Gallego MJ, Garcia-Palacios A, et al. An Internet-Based Self-Help Treatment for Fear of Public Speaking: A Controlled Trial. Cyberpsychology Behavior and Social Networking. Aug 2010;13(4):407-421.

4. Klein B, Cook S. Preferences for e-mental health services amongst an online Australian sample. E-Journal of Applied Psychology. 2010;6(1):28-39.

5. Neal DM, Campbell AJ, Williams LY, Liu Y, Nussbaumer D. "I did not realize so many options are available": Cognitive authority, emerging adults, and e-mental health. Library \& Information Science Research. 2011;33(1):25-33.

6. Esters IG, Cooker PG, Ittenbach RF. Effects of a unit of instruction in mental health on rural adolescents' conceptions of mental illness and attitudes about seeking help. Adolescence. 1998;33(130):469-469-476.

7. Nicholas J. The role of internet technology and social branding in improving the mental health and wellbeing of young people. Perspectives in Public Health. Mar 2010;130(2):86-90.

8. Vega MM, Roland EL. Social Marketing Techniquesfor Public Health Communication: A Review of Syphilis Awareness Campains. Social Marketing Tecnhiqes 2008;32:350. 
9. Dietrich S, Mergl R, Freudenberg P, Althaus D, Hegerl U. Impact of a campaign on the public's attitudes towards depression. Health Education Research. Feb 2010;25(1):135-150.

10. Nicholas J, Oliver K, Lee K, O'Brien M. Help-seeking behaviour and the Internet: An investigation among Australian adolescents Australian e-Journal for the Advancement of Mental Health. 2004;3:1-7.

11. Sharp W, Hargrove DS, Johnson L, Deal WP. Mental Health Education: An Evaluation of a Classroom Based Strategy to Modify Help Seeking for Mental Health Problems. Journal of College Student Development. 2006;47(4):419-438.

12. LimeSurvey [computer program]. Version; 2011.

13. Mohr DC, Siddique J, Ho J, Duffecy J, Jin L, Fokuo JK. Interest in Behavioral and Psychological Treatments Delivered Face-to-Face, by Telephone, and by Internet. Annals of Behavioral Medicine. Aug 2010;40(1):89-98.

14. Tsan JY, Day SX. Personality and gender as predictors of online counseling use. Journal of Technology in Human Services. 2007;25(3):39-55. 
